Polymer Journal, Vol. 39, No. 7, pp. 703-711 (2007)

(C) 2007 The Society of Polymer Science, Japan

\title{
Structure and Properties of Low-Substituted Hydroxypropylcellulose Films and Fibers Regenerated from Aqueous Sodium Hydroxide Solution
}

\author{
Miki KiYose, Eriko Yamamoto, Chihiro Yamane, ${ }^{\dagger}$ \\ Takehiko MIDORIKAWA, and Toshisada TAKAHASHI
}

Faculty of Home Economics, Kobe Women's University, 2-1 Aoyama Higashisuma, Suma-ku, Kobe 654-8585, Japan

(Received January 5, 2007; Accepted April 3, 2007; Published May 22, 2007)

\begin{abstract}
The structure of Low-Substituted Hydroxypropyl Cellulose (LHPC) films obtained from aqueous sodium hydroxide solution by coagulation with aqueous sulfuric acid/sodium sulfate was investigated, mainly by WideAngle X-ray Diffraction (WAXD) and SEM. Both edge-on and through view WAXD patterns of films dried under compression revealed that the (110) planes of the LHPC crystals were preferentially oriented parallel to the film's top surface. The d-spacing of (110) planes of LHPC was larger than that of cellulose II by $9 \%$, indicating that a part of the hydroxyl groups in LHPC crystals were replaced with hydroxypropyl groups. The replacement of the (110) diffraction arc in the edge view by a strong streak at low diffraction angle, and the considerable swelling anisotropy of the films in the thickness direction suggested that water molecules penetrated not only into the amorphous regions but also into the LHPC crystals, bringing about planar fracture along the (110) planes.

A complicated multicellular structure containing pores of widely varying sizes was observed in SEM micrographs of the side surface of freeze-fractured LHPC film. Each cell was surrounded by thin membranes $c a$. $0.1-1.0 \mu \mathrm{m}$ in thickness. The formation of this supramolecular structure and the mechanism of selective uniplanar orientation of (1 110$)$ crystal planes were discussed on the basis of the WAXD patterns, SEM micrographs and fractal analysis.

[doi:10.1295/polymj.PJ2006206]

KEY WORDS Hydroxypropylcellulose / Regenerated Film / Selective Uniplanar Orientation / Anisotropic Swelling / Layer Structure / Wide-Angle X-ray Diffraction / Fractal Aggregation /
\end{abstract}

The reaction between alkali cellulose and carbon disulfide known as the "viscose reaction" has been the basis of the production of rayon, staple fiber, cellophane, sponges and many kinds of related products. Though unfortunately the viscose industry inevitably discharges pollutant sulfur compounds into the environment, cellulose products generated from "viscose" have many useful characteristics, especially a hydrophilic nature, which can not be achieved using most synthetic polymers. Therefore, development of new production systems is becoming increasingly important in the regenerated cellulose fiber industry.

In recent years, there have been many efforts to find new, less-polluting cellulose solvents for producing fibers and films. Cellulose fibers spun from $N$-methyl-morpholine- $N$-oxide solution are expected to be ecologically friendly, since they are produced in a closed process without discharging pollutants. ${ }^{1-4}$

The development of alkali-soluble cellulose is expected to play an important role in the cellulose industry. Numerous studies have been conducted by Asahi Chemical Industries on alkali-soluble cellulose ${ }^{5,6}$ and have led to the successful bench scale production of a new type of cellulose fiber. ${ }^{7}$ In the course of research and development, the mechanism of cellulose dissolution into sodium hydroxide solution was fully understood ${ }^{8}$ followed by the formulation of dissolution techniques. ${ }^{9}$ When this knowledge was applied to the dissolution of cellulose derivatives with a low degree of substitution (DS), the solubility and stability of the dope was greatly improved. For example, the upper limit of polymer concentration increased up to $10 \mathrm{wt} \%$ for cellulose derivatives with $0.1 \mathrm{DS}$, whereas it was only $6 \mathrm{wt} \%$ for the alkali-soluble cellulose.

Low-substituted hydroxypropyl cellulose (LHPC), used for making medicine tablets, has received limited attention in the field of regenerated cellulose, probably because of its rather low solubility in sodium hydroxide solution, thus not producing a suitable dope for fibers and films. However, the dissolution technique used for alkali-soluble cellulose allowed LHPC to dissolve completely in aqueous $\mathrm{NaOH}$. In addition, LHPC still retained the characteristic properties of cellulose II because of its low DS; for example, the crystal structure of LHPC was very similar to that of cellulose II, making LHPC a suitable alternative to regenerated cellulose. Films prepared from LHPC/aq. sodium hydroxide solution were found to have almost the same mechanical properties as those of regenerated cellulose films. ${ }^{10}$ In addition to vying for the com-

${ }^{\dagger}$ To whom correspondence should be addressed (E-mail: yamane@suma.kobe-wu.ac.jp). 
mercial applications of regenerated cellulose, LHPC could also be used in foods in the form of cellulose shaped articles. In this context, edible films have been investigated, e.g., as an alternative to gelatin in pharmaceutical capsules.

In this study, we have examined the structure and properties of LHPC films and fibers regenerated from sodium hydroxide solution in a coagulation bath containing sulfuric acid and sodium sulfate. Mechanisms of gelation, the selective uniplanar orientation behavior of (110) planes during coagulation, the remarkable anisotropic swelling behavior of the coagulated films, and the change in the crystal structure of LHPC on swelling will be discussed based on the results of WAXD and SEM studies.

\section{EXPERIMENTAL}

\section{Materials}

LHPC powders containing $10.8 \%$ hydroxypropyl groups (molecular substitution $\mathrm{MS}=0.33$ ) were kindly supplied by Shinetsu-Kagaku Kogyo Co Ltd.; the sample grade was C-21. Reagent grade carboxymethyl cellulose (CMC), sodium hydroxide, sulfuric acid, and anhydrous sodium sulfate were purchased from Kishida Chemical Co. Ltd. and were used without further purification. Specimen test kits for evaluating the concentration of metal and ammonium ions in water were also purchased from Kishida Chemical Co. Ltd.

\section{Preparation of LHPC Films and Fibers}

An alkali LHPC solution with a polymer concentration of $7 \mathrm{wt} \%$ and an alkali concentration of $8 \mathrm{wt} \%$ was prepared as follows: LHPC powder was dispersed in cold $\left(1-2^{\circ} \mathrm{C}\right)$ water for $2 \mathrm{~h}$. To the LHPC slurry thus obtained, cold $\left(-5--10^{\circ} \mathrm{C}\right) 30 \%$ aqueous sodium hydroxide solution was added, and the mixture was stirred for $3 \mathrm{~h}$ below $0{ }^{\circ} \mathrm{C}$.

A layer of alkali solution of LHPC was applied to a glass plate using a $10 \mathrm{~cm} \times 12 \mathrm{~cm} \times 1 \mathrm{~mm}$ polyvinyl chloride sheet frame. The glass plate carrying this solution was immersed in the coagulation bath containing $10 \%$ sulfuric acid and $20 \%$ sodium sulfate at room temperature, and rinsed repeatedly with deionized water. As-prepared LHPC films (FG film: fresh gel films) were placed between some filter papers and dried under compression of about $0.2 \mathrm{~kg} / \mathrm{cm}^{2}$ at room temperature for one week, during which time the filter papers were exchanged three times. LHPC films thus obtained will hereafter be referred to as PD-LHPC films (PD: press-dried). LHPC fibers were prepared using a test-spinning machine equipped with a stainless steel disk having 100 holes of diameter $0.1 \mathrm{~mm}$. LHPC/CMC (70/30) blend films and fibers were pre- pared from aqueous sodium hydroxide solution in a manner similar to that described above.

\section{Wide-Angle X-ray Diffraction (WAXD)}

Stacked LHPC ribbons $(0.5 \mathrm{~mm}$ wide $\times 10 \mathrm{~mm}$ long $\times 0.7 \mathrm{~mm}$ thick) were used for X-ray measurements. X-Ray diffraction patterns taken perpendicular and parallel (through view and edge view, respectively) to the top surface of LHPC films were recorded on a Rigaku Denki model RAXIS IIC X-ray diffractometer equipped with an imaging plate, operated at $40 \mathrm{kV}$ and $100 \mathrm{~mA}$. Graphite-monochromatic $\mathrm{CuK} \alpha$ radiation was utilized as the $\mathrm{X}$-ray source. Wet films were wrapped with aluminum foil to prevent water from evaporating during the measurements, which gave rise to diffraction rings of aluminum at far higher diffraction angles than those of LHPC.

\section{Scanning Electron Microscopy (SEM)}

Scanning electron microscopic observations of the fracture surface of lyophilized LHPC films were carried out with a Hitachi scanning electron microscope (S-2250N). Samples were coated with Au by vacuum evaporation before observation.

\section{RESULTS AND DISCUSSION}

\section{WAXD Patterns of Regenerated LHPC Films}

LHPC with a degree of substitution of $10.8 \%$ was readily soluble in aqueous sodium hydroxide solution, forming a clear solution under appropriate conditions. PD-LHPC films, prepared from the solution and dried under compression to prevent drying shrinkage, were subjected to WAXD measurements. Because of its low degree of substitution, LHPC still maintained the characteristics of cellulose II, i.e., the original and regenerated LHPC gave WAXD patterns similar to that of cellulose II, though the crystallinity of LHPC was lower than that of cellulose II and the $\mathrm{d}$-spacing of the (110) plane of LHPC was slightly larger than that of cellulose II, as discussed below. The three main diffraction peaks of LHPC crystal were here labeled (1 $\overline{1} 0),(110)$ and (020), respectively, in order of increasing diffraction angles, corresponding to cellulose II peaks. ${ }^{11}$ The orientation of the (110) plane in these films was estimated from the WAXD patterns obtained by X-ray irradiation parallel and perpendicular to the top surface of the films. These WAXD patterns will hereafter be referred to as the EV-WAXD (Edge View) pattern and TVWAXD (Through View) pattern, respectively.

Figure 1(a) and (b) are EV-WAXD and TVWAXD patterns, respectively, of the PD-LHPC film. The EV-WAXD pattern showed the (110) arc on the equator and the (110) arc on the meridian, with a dif- 


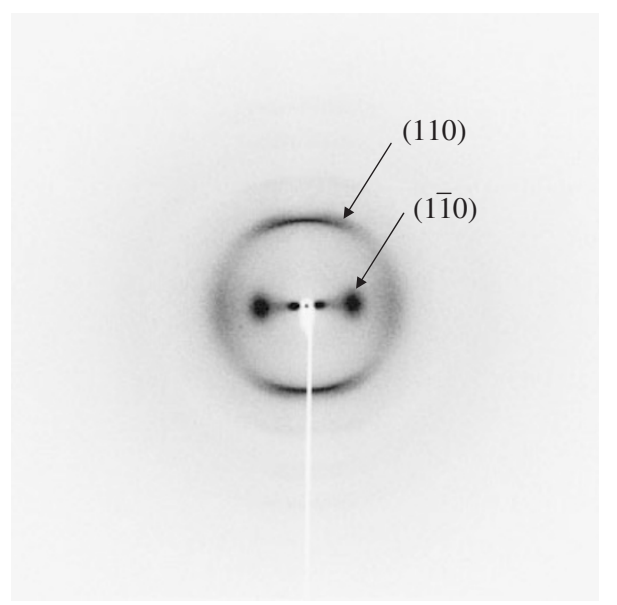

(a) Edge View

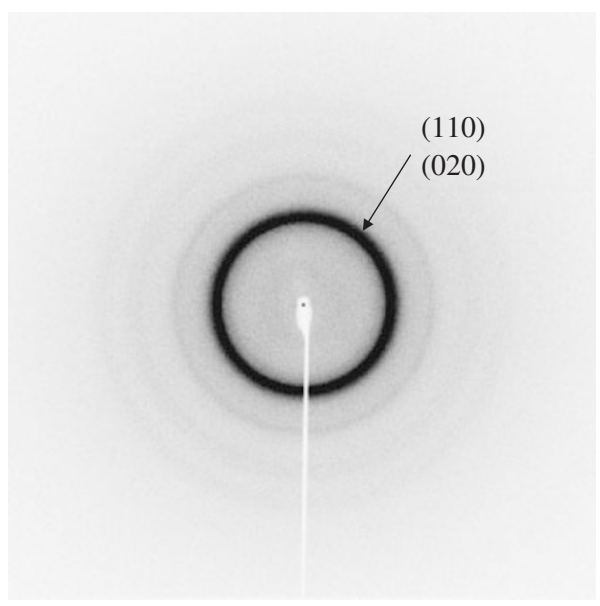

(b) Through View

Figure 1. WAXD patterns of PD-LHPC film: (a) Edge View (EV) and (b) Through View (TV).

fuse scattering arc (probably originating from amorphous chains) as an intensity maximum on the equator. On the other hand, the TV-WAXD pattern was missing the (110) reflection, whereas rather strong (110) and (020) doublet rings were visible. Thus, it could be concluded that both the (110) planes of the LHPC crystals and the amorphous chains were preferentially oriented parallel to the film surface. Taking into account the considerable swelling anisotropy in the thickness direction, it was deduced that most of the amorphous chains lay between the (110) surfaces of LHPC crystallites. The swelling behavior of the film will be discussed later.

The WAXD patterns in Figure 1 were similar to those of cellulose II film regenerated from viscose in a coagulation-regeneration bath containing a high concentration of sulfates, ${ }^{12}$ though the diffuse scattering arc originating from the amorphous chains was not as clear as in the WAXD pattern of the regenerated LHPC films.

An attempt was made to identify the differences between the crystal structures of LHPC and cellulose II.

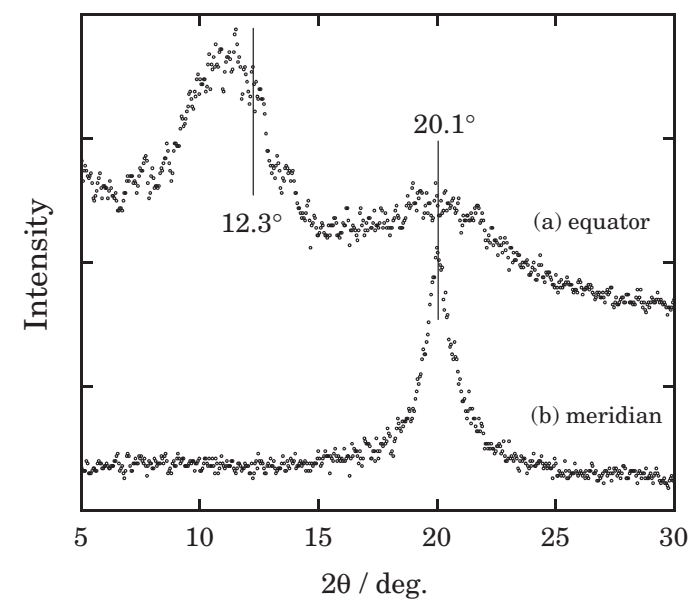

Figure 2. WAXD intensity distribution curves measured along the equator (a) and the meridian (b) of the EV-WAXD pattern of PD-LHPC film.

Figure 2 shows the WAXD intensity distribution curves measured along the equator and the meridian of the EV-WAXD pattern (Figure 1(a)). The equatorial intensity distribution curve showed the (110) peak of LHPC at $2 \theta=c a \cdot 11^{\circ}$, whereas the (110) peak of cellulose II is at $2 \theta=12.3^{\circ}$. This indicated that the $\mathrm{d}$-spacing of the (110) planes of LHPC was $c a$. 9\% larger than in cellulose II. On the other hand, the $\mathrm{d}$-spacing of the (110) plane of LHPC was the same as that of cellulose II, $2 \theta=20.1^{\circ}$. This result indicated that the reaction of propylene oxide with cellulose II took place between the (110) planes of LHPC crystals where the hydroxyl groups were located, and that some of these groups were replaced with hydroxypropyl groups, expanding the d-spacing of (110) plane.

\section{WAXD Patterns of Swollen LHPC Films}

$X$-Ray studies of PD-LHPC films swollen in water (PD-RS-LHPC) (RS: Re-Swelled) yielded interesting results, discussed below in subsections (a)-(d).

(a) Disappearance of the (110) arc in EV-WAXD pattern. Figure 3 shows the EV- and TV-WAXD patterns of PD-RS-LHPC film. The EV-WAXD pattern showed no (110) arc derived from the LHPC crystal structure after the film was swollen in water, although a rather sharp (110) arc was still present near the meridian, indicating that water molecules had permeated into the LHPC crystal. It seemed reasonable to assume that the penetration of water into the crystalline regions of LHPC brought about planar fracture along the $(1 \overline{1} 0)$ planes to produce planar crystals, whose size in the (110) direction was considerably smaller than that in the (110) direction.

(b) Appearance of a strong streak along the equator at low diffraction angles. After swelling, the EVWAXD pattern shown in Figure 3(a) had a strong 


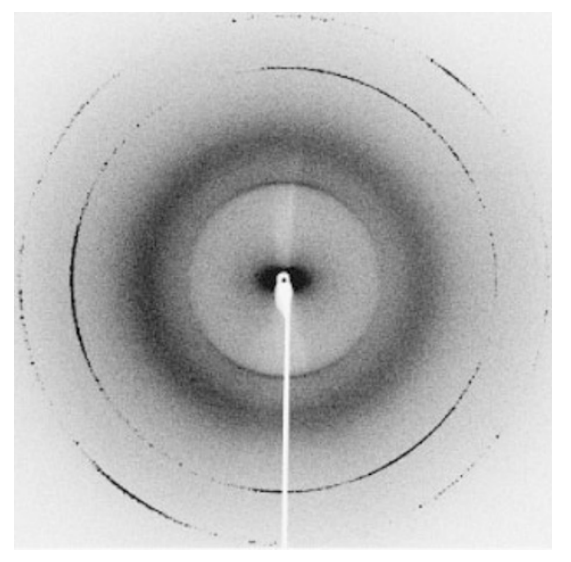

(a) Edge View

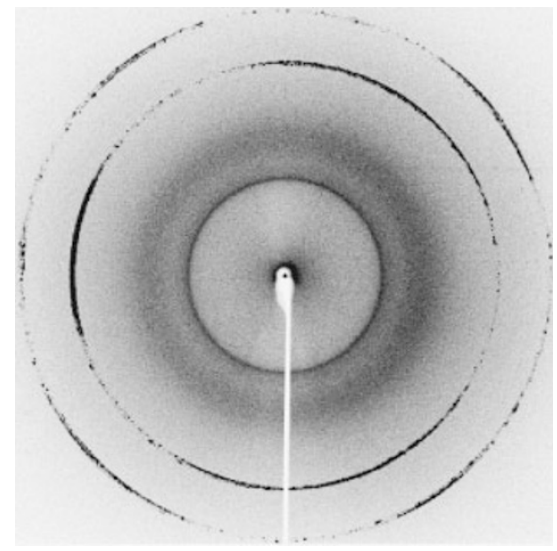

(b) Through View

Figure 3. WAXD patterns of PD-RS-LHPC film: (a) Edge View (EV) and (b) Through View (TV). The two outer rings were the diffractions of aluminum; the wet film was wrapped with aluminum foil in order to prevent evaporation of water during the measurements.

streak along the equator at low diffraction angles $\left(2 \theta<c a .10^{\circ}\right)$ in place of the $(1 \overline{1} 0)$ arc. This result could also be explained by the planar fracture of LHPC crystals along the (110) planes resulting in different (110) d-spacings, which were larger than that of the original dry LHPC crystal. X-Ray studies of regenerated cellulose II films, however, showed that the (110) arc did not disappear on swelling.

(c) Disappearance of the diffuse arc originating from oriented amorphous chains. The diffuse scattering arc near the equator originating from oriented amorphous chains disappeared completely on swelling, as shown in Figure 3(a). Water molecules had also penetrated into the amorphous regions of PDLHPC, expanding the distance between amorphous chains and disordering the orientations parallel to the film surface.

(d) Spreading of the (110) arc over the azimuthal angle. Although the (110) arc disappeared, a rather sharp (110) arc, spread over the azimuthal angle, was still observed in Figure 3(a), indicating that the
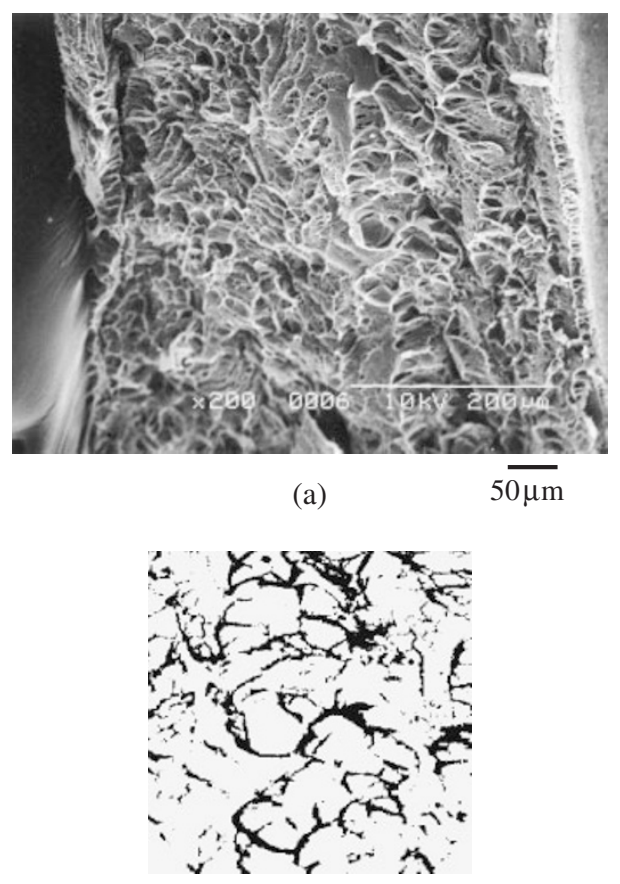

(b)

Figure 4. (a) SEM micrograph of side surface of LHPC fresh gel film, freeze fractured and lyophilized; (b) transferred high contrast black-and-white image of (a).

planar orientation of LHPC crystallites became disordered to some extent upon swelling.

\section{Scanning Electron Microscopy}

(1) Side surface of the freeze dried fresh gel film. Fresh LHPC gel films were freeze fractured and lyophilized. Figure 4(a) is the SEM micrograph of the freeze fractured side surface of a LHPC fresh gel film showing a complicated multicellular structure. A high contrast black and white image corresponding to Figure 4(a) is shown in Figure 4(b). Many pores with varying diameters, $c a .0 .5-15 \mu \mathrm{m}$, were visible in the SEM micrographs, and each cell was surrounded by thin membranes $c a .0 .2-1.0 \mu \mathrm{m}$ in thickness. These thin membranes, which constituted the three-dimensional network in the fresh gel films, will hereafter be referred to as "elemental thin membranes." The multicellular arrangement of pores of widely varying size appeared to be a fractal structure, characteristic of fractal structures formed by the diffusion limited aggregation of particles. ${ }^{13,14}$ Assuming that the SEM micrograph reflected the original gel structure, the fractal dimension for the multicellular structure could be estimated as follows.

The SEM micrograph shown in Figure 4(a) was converted to a high contrast black-and-white image (Figure 4(b)) for the estimation of fractal dimension using a box-counting technique. ${ }^{15}$ First, $\log N(d)$ was plotted against $\log d$, where $N(d)$ is the number of 


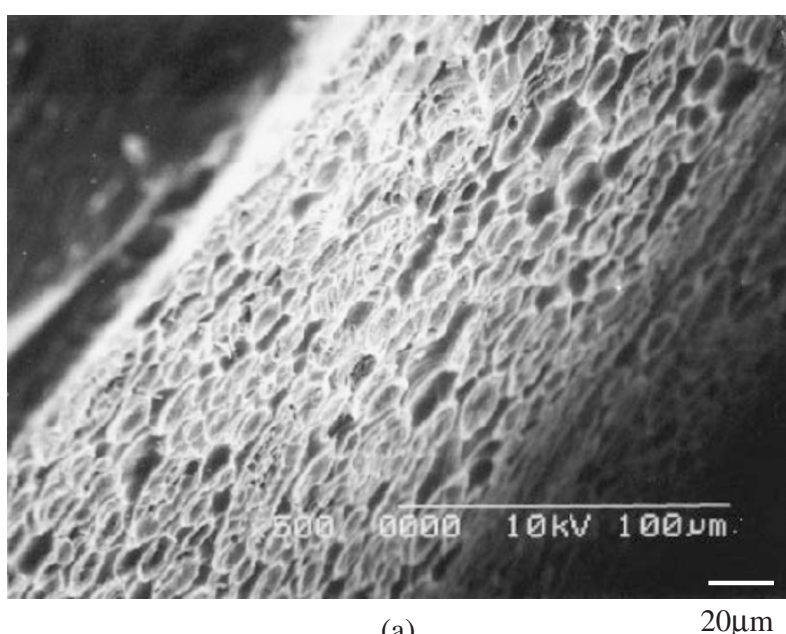

(a)

$20 \mu \mathrm{m}$

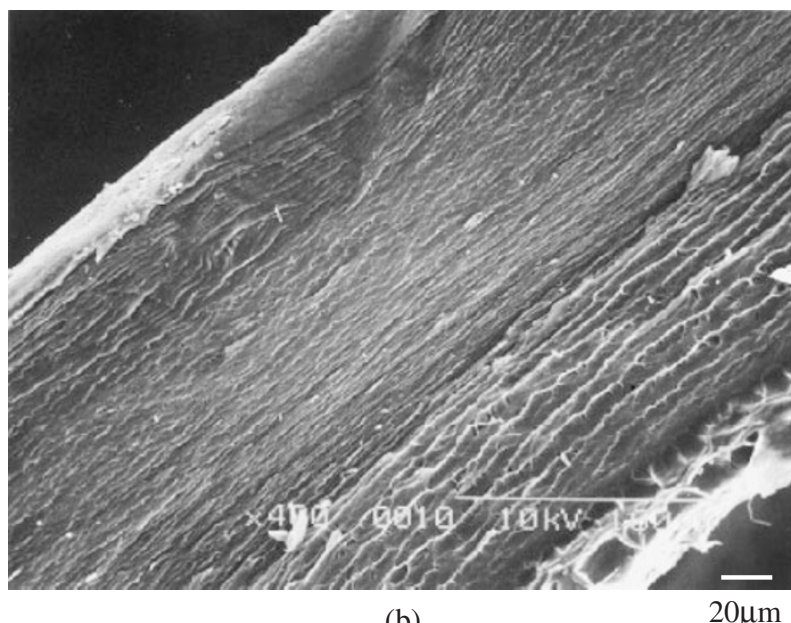

(b)

$20 \mu \mathrm{m}$

Figure 5. SEM micrographs of side surface of PD-RS-LHPC film, freeze fractured and lyophilized: (a), re-swollen in water to five times the original volume; (b), twice the original volume.

boxes of side $d(\mathrm{~cm})$ which contained a part of the black areas in Figure 4(b). From the slope of the $\log N(D)$ vs. $\log d$ plot, the fractal dimension $D$ was estimated to be 1.33 . The gelation mechanism will be discussed later on in this paper.

(2) Freeze fractured side surface of the swollen PDLHPC film. PD-LHPC film was re-swollen in water to five or two times its original volume at $25^{\circ} \mathrm{C}$ for $3 \mathrm{~h}$ and lyophilized. Figure 5 shows the SEM micrographs of the freeze fractured side surface of this PD-RS-LHPC film. For the five-fold swollen film, ellipsoidal multicellular structures were observed in Figure 5(a); the long axis of the ellipsoidal pore lay parallel to the top surface of the film. This finding could best be explained by assuming that the original three-dimensional network composed of elemental thin membranes collapsed during compression and was dried without further alteration. For the two-fold swollen film, stacked layer structures (probably originating from the multicellular structures) were observed in Figure 5(b).
Mechanism of Gelation and of the Selective Uniplanar Orientation of (110) Planes

The mechanism of gelation and the selective uniplanar orientation of (110) crystal planes will now be discussed in light of the WAXD patterns, SEM micrographs and fractal analysis.

Gelation. The molecular structure of LHPC was very similar to that of cellulose II, except that some of the hydroxyl groups were replaced with hydroxypropyl groups in LHPC, even in the crystallites. Since the hydroxyl groups and hydroxypropyl groups were located on the side surfaces of the glucopyranose rings, the area around the side surface was hydrophilic, whereas the area around the top surface of these rings was hydrophobic, as discussed before by Takahashi et al. ${ }^{12,16}$

It was possible that the three-dimensional network composed of the elemental thin membranes was formed according to steps (i) to (iii) below during the coagulation process.

(i) In the first stage of coagulation from aqueous alkali solution, it was reasonable to assume that LHPC molecules aggregated side by side, with glucopyranose rings stacking by hydrophobic interactions, to form a layer structure as shown schematically in Figure 6(a). The existence of this layer structure necessary for this hypothesis correlates well with the concept of glucan sheet formation being the first step of crystallization, as previously proposed in various studies on dye-altered cellulose. ${ }^{17,18}$ Energetics studies using the MM3 molecular mechanics program indicated that van der Waals-associated molecular sheets corresponding to these layer structures had a far lower potential energy than that of hydrogen bonded molecular sheets in aqueous media. ${ }^{19}$ It was concluded that van der Waals-associated molecular sheets were the most likely structures formed in the initial step of crystallization. ${ }^{19}$ Additionally, Hayashi ${ }^{20}$ and Hermans ${ }^{21}$ have identified such layer structures (naming them Plane Lattice Structures or Sheet-like Structures) as the basic features of regenerated cellulose.

(ii) As the coagulation proceeded, several layer structures stacked progressively to form thin planar LHPC crystals (Figure 6(b)), which further aggregated to form "precursor elemental thin membranes" incorporating amorphous chains as shown in Figure 6(c). Thus, (110) planes were naturally oriented parallel to the surface of the precursor elemental thin membranes, as shown schematically in Figure 6(c). At this stage the three-dimensional network would probably not have been formed yet.

(iii) These precursor elemental thin membranes randomly dispersed in solution stuck together upon contact to form the three-dimensional network shown in Figure 6(d). 
(a) |ルாルाாルாルா

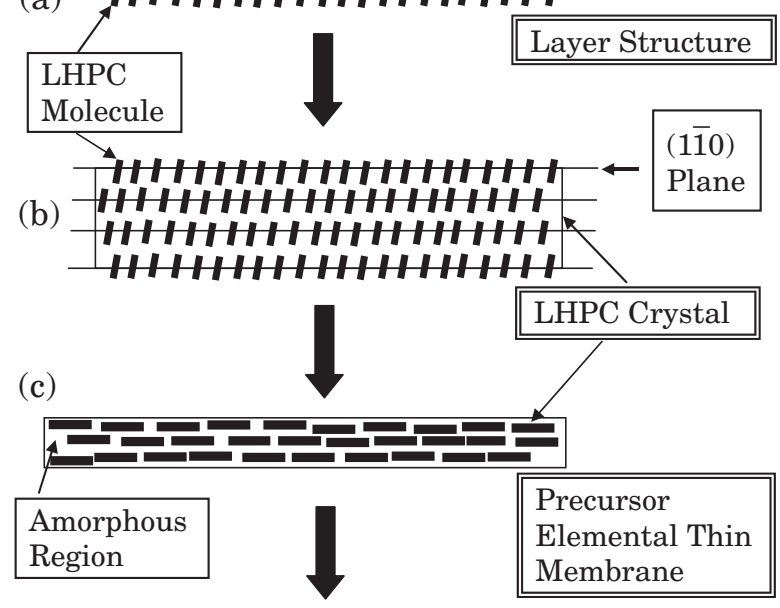

(d)

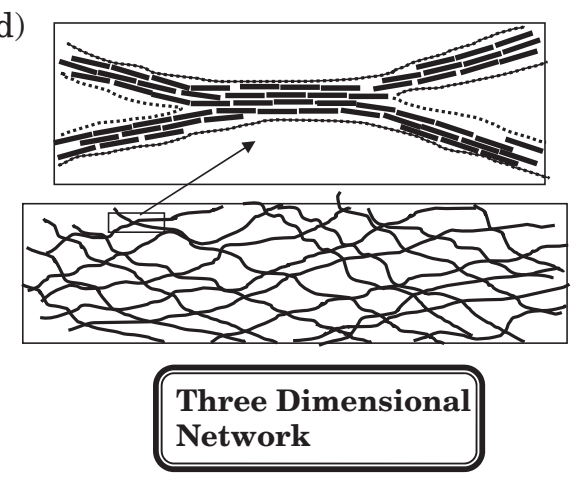

Figure 6. Schematic model representing structural formation during the coagulation process.

On the basis of computer simulations, Kolb et al. have reported that the fractal dimension for the diffusion limited cluster-cluster aggregation of particles was $1.38,{ }^{13}$ whereas the fractal dimension for the diffusion limited aggregation of single particles was 1.68. ${ }^{14}$ The first model, diffusion limited cluster-cluster aggregation, started with an assembly of particles which stuck together upon contact to form rigid clusters. Clusters thus formed could diffuse along with particles and continue to grow by aggregation when they met other clusters or particles. The calculations performed demonstrated that a cluster of $N$ particles moved like a Brownian particle of mass $N$ (probability of moving $\propto 1 / \sqrt{N}$ ). The second model, diffusion limited aggregation of single particles, started with a seed particle fixed at the origin of a lattice, while another particle was allowed to walk at random (i.e., diffuse) from far away until it arrived at the seed particle, when it stopped. Successive particles were then launched and allowed diffuse until each one arrived at any occupied site. In this manner, large clusters were formed.

As described above, the fractal dimension was estimated to be 1.33 for the freeze-fractured LHPC fresh gel film. This value was close to $D=1.38$, the fractal dimension for the first model, the diffusion limited cluster-cluster aggregation model. If it is assumed that aggregates of the "precursor elemental thin membranes" can be treated as particles or clusters, then it may be that diffusion limited cluster-cluster aggregation plays an important role during the gelation of LHPC.

As shown by computer simulations, the fractal structures formed through cluster-cluster aggregation were characterized by voids with widely varying diameters. Similarly, lyophilized fresh gels contained pores of various sizes, as shown in Figure 4(a). In addition, the SEM micrographs seemed to reveal self-assembling aspects characteristic of fractal structures.

In recent years, structural formations and supramolecular assemblies have been subjected to fractal analysis as shown in following examples: the fractal dimension for aggregates of gel microspheres of itpoly(methyl methacrylate) from xylene solution was calculated to be about $1.5,{ }^{22}$ implying that the particles formed over time and collided with each other to form the fractal structures; the temperature dependence of the fractal dimension of aggregates of polystyrene latex particles revealed the mechanism of aggregation in aqueous electrolyte solution, ${ }^{23}$ and the fractal dimension of the microporous structure of a polyurethane membrane was determined by the boxcounting technique. ${ }^{24}$

Selective uniplanar orientation. According to earlier papers, the selective uniplanar orientation seen in cellulose II could be explained by a mechanism involving the rotation of its crystals. The absorbed water located on the (1 $\overline{1} 0)$ surface of cellulose II crystals plasticized the planar cellulose crystallites, allowing them to glide over each other's (110) surfaces. ${ }^{12,25}$ It was reasonable to assume that a similar phenomenon occurred during the formation of the elemental thin membranes from their layer components.

Attempts were made to elucidate the mechanism of selective uniplanar orientation from the WAXD patterns and SEM micrographs of the freeze fractured side surface of the fresh LHPC gel film.

The EV- and TV-WAXD patterns of the fresh LHPC gel film are shown in Figure 7. A rather sharp (110) ring, a strong diffuse scattering ring due to water and ellipsoidal central scattering were visible in the EV-WAXD pattern. The SEM micrograph (Figure 4) and the WAXD patterns (Figure 7) indicated that the fresh gel film was composed of a three-dimensional network of elemental thin membranes containing planar LHPC crystals. If it could be assumed that the (110) planes were oriented parallel to the elemental thin membranes, then a selective uniplanar orientation of the (1110) crystal planes parallel to the film's top surface was obtained when the fresh gel films were dried under compression in order not to alter 


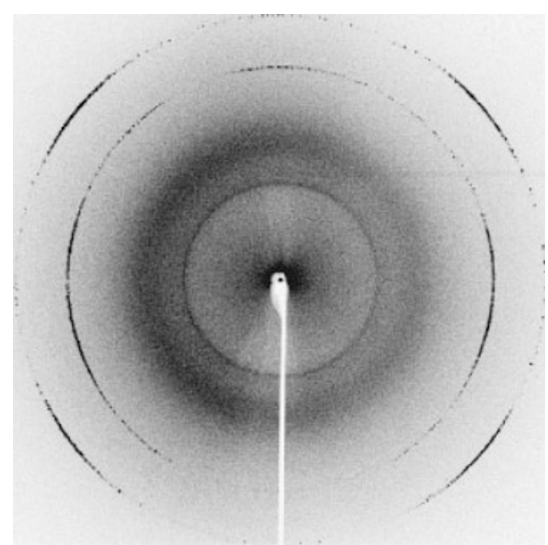

(a) Edge View

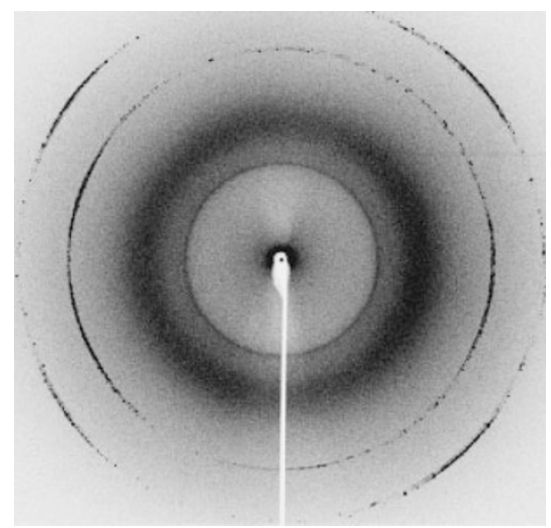

(b) Through View

Figure 7. WAXD patterns of fresh LHPC gel film: (a) Edge View (EV), and (b) Through View (TV). The two outer rings were the diffractions of aluminum (see Figure 3 legend).

their rectangular shape or size. On the other hand, when the fresh gel films were dried freely without compression, allowing dimensional changes, LHPC films with randomly oriented (1110) planes were formed. This dimensional instability of the fresh LHPC gel films possibly derived from their low crystallinity and absorption of water even in the crystalline regions.

In contrast, the selective uniplanar orientation of (110) planes still occurred when fresh cellulose II gel films regenerated from viscose in coagulation baths containing a high concentration of sulfates were dried freely. ${ }^{12}$

The fringed micelle-type structure model has been widely accepted for gels of some polysaccharides. The foregoing SEM and WAXD studies showed that LHPC gel films were constituted of thin membranes having a selective uniplanar orientation of (11) planes. This structural model seemed more appropriate than the fringed micelle type structure for PDLHPC films, because there did not seem to be any routes from the fringed micelle structure to the multicellular structure like the one shown in Figures 4 and
5. The proposed gelation mechanism was consistent with the results of X-ray and SEM studies.

\section{Re-Swelling of the PD-LHPC Films}

Regenerated PD-LHPC film absorbed about 420$435 \mathrm{wt} \%$ of water at room temperature, whereas regenerated cellulose II films absorbed about $200 \%$ of water. This result could be attributed to the lower crystallinity of LHPC. Furthermore, water could penetrate not only into the amorphous region but also into the crystalline region of LHPC, as the WAXD pattern of PD-RS-LHPC film shows in Figure 3.

Considerable swelling anisotropy in the thickness direction was found by Takahashi in cellulose films regenerated from viscose. ${ }^{12}$ PD-LHPC films also revealed a considerable swelling anisotropy. For example, the side length of a rectangular PD-LHPC film regenerated in a bath consisting of $10 \%$ sulfuric acid and $20 \%$ sodium sulfate remained almost unchanged on swelling, whereas the film itself swelled in thickness by $400 \%$.

This anisotropic swelling behavior of LHPC films could readily be explained on the basis of the WAXD and SEM observations. LHPC films were composed of stacked thin membranes, where thin crystallites and oriented amorphous chains were alternatively stacked as shown in Figure 8. Water penetrated not only into the crystals and the amorphous regions, but also into the planar spaces between the thin membranes constituting the LHPC film, as discussed below.

(1) First, as distinct from cellulose II, water could penetrate into the PD-LHPC crystalline region as shown in Scheme (b) of Figure 8, and it was reasonable to assume that it was retained as water of hydration between the $(1 \overline{1} 0)$ crystal planes. As a result, the (110) reflection disappeared, and a strong equatorial streak appeared at lower diffraction angle as shown in Figure 3(a).

(2) Needless to say, water penetrated into the oriented amorphous chain segments, as depicted in Scheme (b) of Figure 8, bringing about an increase in thickness of the thin membranes upon swelling. As a result, the diffuse scattering arc near the equator (Figure 1(a)) originating from the amorphous chains disappeared on swelling (Figure 3(a)) and the planar orientation of crystals was disturbed, defined by widely spreading (110) arc over azimuthal angle (Figure 3(a)).

(3) As SEM micrographs showed, regenerated PDLHPC was composed of stacked thin membranes. It was possible to conclude that water permeated into the planar spaces between these membranes in addition to the regions described in (1) and (2), and consequently, the previously crushed multicellular structure reformed with ellipsoidal voids, as shown in Figure 5(a). 
(a)

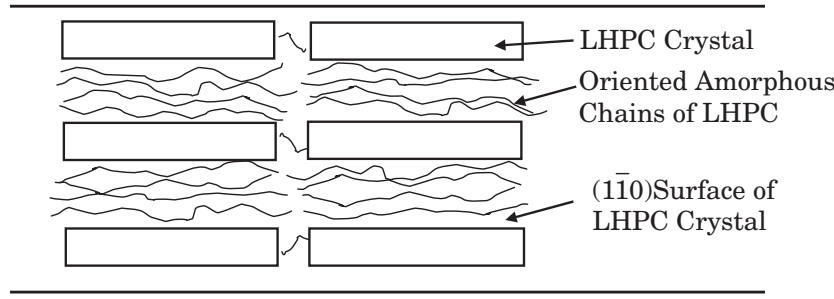

(b) Swelling in Water

Film Surface

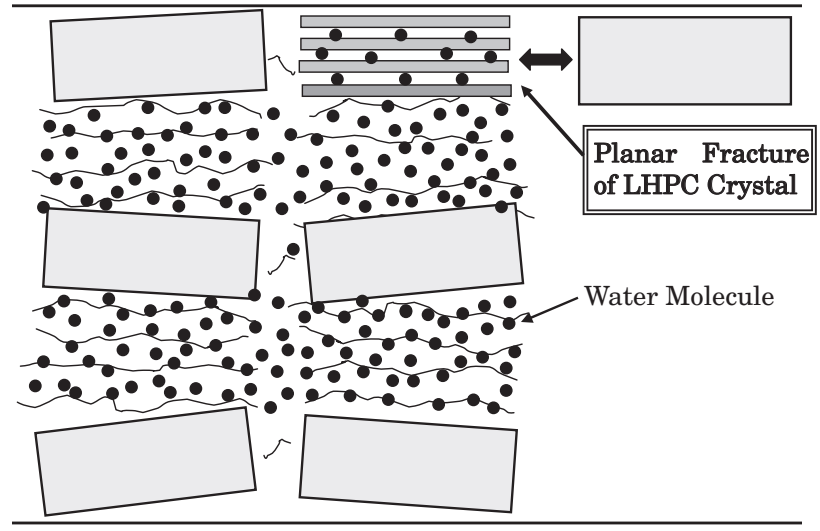

Figure 8. Schematic model representing the anisotropic reswelling of PD-LHPC film.

\section{Structure of Regenerated LHPC Fiber}

A frozen LHPC gel fiber of $c a .1 \mathrm{~mm}$ diameter was snapped and then lyophilized. Figure 9 shows SEM micrographs of the freeze fractured cross-section of this fiber. It was interesting to note that the thin membranes were formed parallel to the fiber surface in the outer region of the fiber, whereas they were oriented randomly near the central region of the fiber.

When an aqueous sodium hydroxide solution of LHPC was extruded through a capillary into a coagulation bath consisting of $10 \%$ sulfuric acid and $20 \%$ sodium sulfate, an aqueous solution of sodium sulfate formed by neutralization diffused out in directions perpendicular to the fiber surface.

As described above, thin membranes with their (110) planes oriented parallel to the surface were formed in the direction of flow of aqueous sodium sulfate solution. This result strongly suggested that the (110) planes themselves were oriented parallel to the fiber surface. In fact, Takahashi has confirmed using the micro-WAXD technique that the (110) planes were oriented parallel to the surface of cellulose model fibers regenerated from viscose in a bath containing sulfuric acid and sulfates. ${ }^{26}$

Structure and Some Functions of LHPC/CMC (carboxymethyl cellulose) Blend Films and Fibers

The structure and certain functions of LHPC/CMC

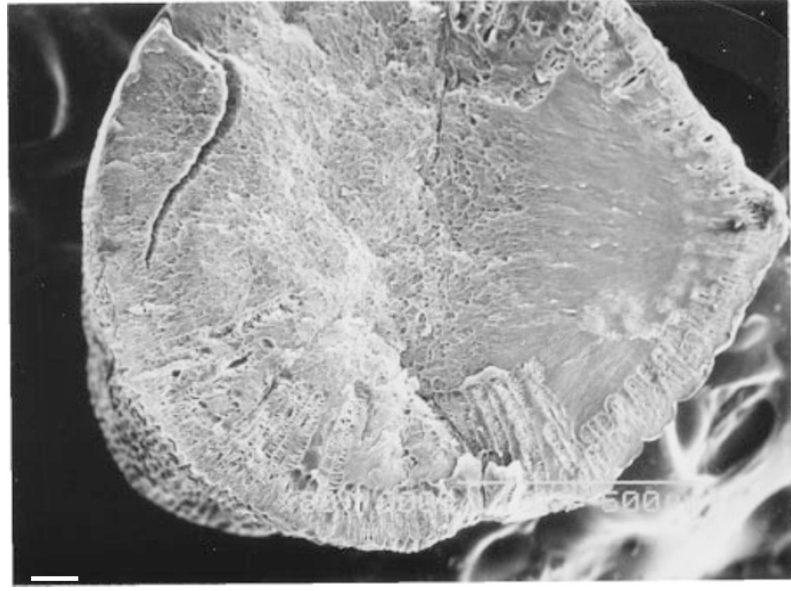

$100 \mu \mathrm{m}$

(a)

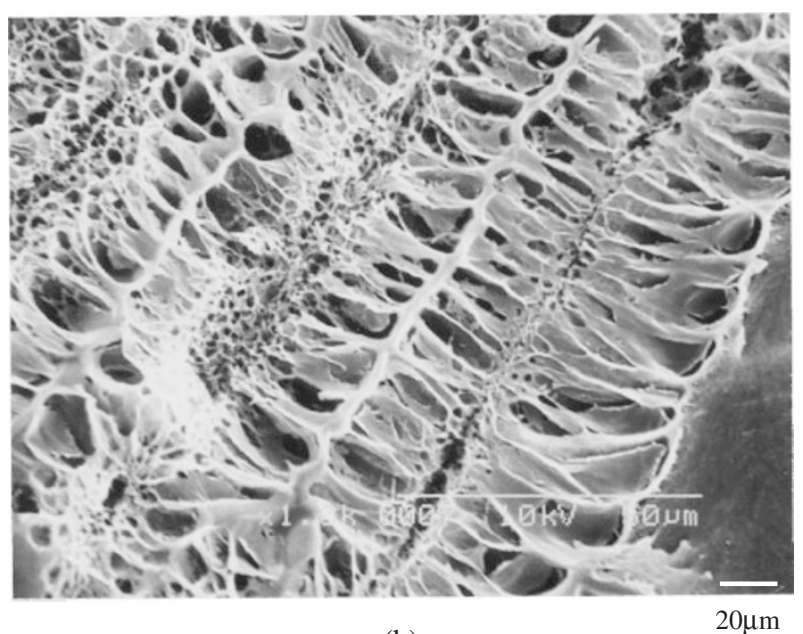

(b)

$20 \mu \mathrm{m}$

Figure 9. SEM micrographs of cross-sectional view of LHPC fiber, freeze fractured and lyophilized.

(carboxymethyl cellulose) blend films and fibers prepared from sodium hydroxide solution were also studied. PD-LHPC/CMC blend films containing 30\% CMC gave WAXD patterns similar to those of LHPC. It was confirmed by a simple testing paper method that they had a high capacity to absorb metal ions such as $\mathrm{Cu}^{2+}$ and $\mathrm{Zn}^{2+}$, and ammonium ions. Complexes of the blend with copper or zinc ions also exhibited considerable antibacterial activities. These results will be reported and discussed in detail elsewhere.

\section{CONCLUSIONS}

The structure and properties of LHPC and LHPC/ $\mathrm{CMC}$ blend films and fibers regenerated from aqueous sodium hydroxide solution in a bath containing sulfuric acid and sodium sulfate were studied, mainly using WAXD and SEM techniques. The following results were obtained:

1) The d-spacing of the (110) planes of LHPC was 
larger than that of cellulose II by $9 \%$, indicating that in LHPC crystals, a part of the hydroxyl groups had been replaced with hydroxypropyl groups.

2) The (110) planes of LHPC crystals and the amorphous chains were preferentially oriented parallel to the film surface of LHPC films dried under compression.

3) Regenerated LHPC films originally dried under compression revealed considerable swelling anisotropy in the thickness direction. Water molecules penetrated not only into the amorphous region but also into the LHPC crystals, bringing about planar fracture along the (110) planes.

4) A complicated multicellular structure containing pores of widely varying size was observed in the SEM micrograph of the side surface of the freezefractured LHPC film. Each cell was surrounded by thin membranes $c a$. $0.2-1.0 \mu \mathrm{m}$ in thickness.

5) The mechanism of gelation and the selective uniplanar orientation of (110) crystal planes was discussed in light of WAXD patterns, SEM micrographs and fractal analysis.

LHPC and LHPC/CMC blend films may be expected to be used widely as new functional materials. The structure and properties of LHPC and related compounds are technologically and scientifically important.

\section{REFERENCES}

1. a) D. L. Jhonson, Brit. Patent 1144048 (1969).

b) D. L. Jhonson, U. S. Patent 3447939, 3508941 (1970).

2. H. Chanzy, A. Peguy, S. Chaunis, and P. Monzie, J. Polym. Sci., Polym. Phys. Ed., 18, 1137 (1980).

3. H. Chanzy, S. Nawrot, A. Peguy, P. Smith, and J. Chevalier, J. Polym. Sci., Polym. Phys. Ed., 20, 1909 (1982).

4. H. Land, I. Laskowski, B. Lukanoff, H. Schleicher, H. Mertel, H. Franz, and E. Taeger, Cellul. Chem. Technol.,
20, 289 (1986).

5. K. Kamide, K. Okajima, and K. Kowsaka, Polym. J., 24, 71 (1992).

6. T. Yamashiki, K. Kamide, K. Okajima, K. Kowsaka, T. Matsui, and H. Fukase, Polym. J., 20, 447 (1988).

7. C. Yamane, M. Mori, M. Saito, and K. Okajima, Polym. J., 28, 1039 (1996).

8. H. Yamada, K. Kowsaka, T. Matsui, K. Okajima, and K. Kamide, Cellul. Chem. Technol., 26, 141 (1992).

9. C. Yamane, M. Saito, and K. Okajima, Sen'i Gakkaishi, 52, 310 (1996).

10. S. Tanioka, Convertech, No. 4, 8 (2002).

11. P. Langan, Y. Nishiyama, and H. Chanzy, Biomacromolecules, 2, 410 (2001).

12. T. Takahashi, Sen'i Gakkaishi, 25, 80 (1969).

13. M. Kolb, R. Botet, and R. Jullien, Phys. Rev. Lett., 51, 1123 (1983).

14. T. A. Witten and L. M. Sander, Phys. Rev. B: Condens. Matter Mater. Phys., 27, 5986 (1983).

15. S. Ishimura, "Fractal Mathematics," 246, Tokyo-Tosho, 1990.

16. C. Yamane, T. Aoyagy, M. Ago, K. Sato, K. Okajima, and T. Takahashi, Polym. J., 38, 819 (2006).

17. A. Kai and T. Koseki, Macromol. Chem., 186, 2609 (1985).

18. R. Malcolm Brown Jr, C. H. Haigler, and K. Cooper, Science, 218, 1141 (1982).

19. S. K. Cousins and R. Malcolm Brown Jr, Polymer, 36, 3885 (1995).

20. J. Hayashi, J. Masuda, and Y. Watanabe, Nippon Kagaku Kaishi, 5, 948 (1974).

21. P. H. Hermans, J. Polym. Sci., 4, 145 (1949).

22. T. Takahashi, K. Kojima, and T. Maegawa, Polymer, 40, 3301 (1999).

23. S. N. Yao, P. W. Zhu, and D. H. Napper, J. Colloid Interface Sci., 174, 162 (1995).

24. M. Enomoto, K. Suehiro, Y. Muraoka, K. Inoue, and M. Sumita, Text. Res. J., 67, 601 (1997).

25. M. Masuo, S. Nomura, and H. Kawai, J. Polym. Sci., Polym. Phys. Ed., 11, 2057 (1973).

26. T. Takahashi, Sen'i Gakkaishi, 25, 122 (1969). 